\title{
High costs as a slow down factor of thoracoscopic lobectomy development in Poland - an institutional experience
}

\author{
Cezary Piwkowski ${ }^{1}$, Piotr Gabryel ${ }^{1}$, Bartłomiej Gałęcki ${ }^{1}$, Magdalena Roszak ${ }^{2}$, Wojciech Dyszkiewicz ${ }^{1}$ \\ 1Department of Thoracic Surgery, Poznan University of Medical Sciences, Poland \\ 2Department of Computer Science and Statistics, Poznan University of Medical Sciences, Poland
}

Videosurgery Miniinv (e-pub, ahead of print) DOI: $10.5114 /$ wiitm.2011.35633

\begin{abstract}
Introduction: Thoracoscopic (VATS) lobectomy after a decade of criticism is nowadays considered as a technically feasible, safe and oncologically proper operation. This approach has some advantages over conventional thoracotomy like: less postoperative pain, shorter hospitalization, fewer postoperative complications, better tolerance of adjuvant chemotherapy with comparable long-term survival rate. The VATS lobectomy is now generally accepted as an important alternative to open lobectomy in early-stage lung cancer.

Aim: In the study we analyzed all aspects of introducing video-assisted thoracoscopic surgery (VATS) lobectomy in our institution with special consideration of the costs of the procedure as a potential limiting factor of its widespread development.

Material and methods: The data of 212 consecutive patients with early stage lung cancer operated on during 20082011 were selected and analyzed. One hundred and eight patients underwent VATS lobectomy (VATS group) and 104 patients antero-lateral thoracotomy (thoracotomy group). Perioperative outcomes including operating time, blood loss during surgery, postoperative complication rate, length of hospital stay, and duration of chest tube drainage were assessed. The cost evaluation included: all direct theater costs, daily hospital costs, intensive care costs, pharmacy and disposable costs with special consideration of stapling device costs.

Results: The mean hospital stay after VATS lobectomy was significantly shorter than after thoracotomy, mean 7 days $v s .10$ days ( $p$ < 0.0012). The complication rate and ICU admission rate were almost twice as high after thoracotomy than after VATS and were $46 \%$ vs. 23\% ( $p<0.0006)$ and $42 \%$ vs. $22 \%(p<0.0027)$ respectively. Cost analysis showed significantly higher total costs of VATS lobectomy than after thoracotomy (median $€ 2445$ vs. €2047). Considerably higher theater costs for VATS compared to thoracotomy, median $€ 1395 \mathrm{vs}$. $€ 479$, were caused mainly by endostapler costs, median $€ 1069$ vs. $€ 161$. Significantly higher hospital costs and ICU costs after thoracotomy did not compensate high theater costs of VATS lobectomy.

Conclusions: In Polish financial reality and potentially in other middle-income countries significantly higher costs of the procedure can limit widespread introduction of VATS lobectomy in clinical practice.
\end{abstract}

Key words: lobectomy, lung cancer, minimally invasive surgery, video-assisted thoracoscopic surgery, health economics.

\section{Introduction}

Worldwide, lung cancer is the leading cause of cancer death for both men and women and surgery is the most effective therapy for these patients [1, 2]. Thoracoscopic lobectomy after a decade of criticism is nowadays considered as a technically feasible, safe 
and oncologically proper operation [3]. Video-assisted thoracoscopic surgery (VATS) is related to less local injury and is now generally accepted as an important alternative to open lobectomy in early-stage lung cancer [4-6]. However, only a few papers have specifically addressed the economic impact of a VATS approach and all these papers came from countries with high financial income [7-9]. This may reflect the economic factor of this procedure as a very important issue which can influence the widespread introduction of a VATS lobectomy program in clinical practice.

In this study we present our experience of introducing a VATS lobectomy program with special consideration of the financial aspect of the procedure in Polish economic reality, which seems to be comparable to other middle-income countries.

\section{Aim}

Analysis of all aspects of introducing VATS lobectomy as a new procedure with special consideration of the costs of the procedure as a potential limiting factor of its widespread development.

\section{Material and methods}

Between January 2008 and April 2011 at the Department of Thoracic Surgery of Poznan University of Medical Sciences 720 patients underwent lobectomy due to lung cancer. After approval by the MedicalMedical University Institutional Review Board, the data of 212 patients with early stage lung cancer were selected and analyzed in this study. To minimize the risk of selection bias in our paper we selected the patients according to the T status. It was our matching feature. The entry criteria included all patients with tumors less than $5 \mathrm{~cm}$, without infiltration of the bronchial tree visible at bronchoscopy or chest wall invasion and without mediastinal nodal involvement. The same indications were used for VATS lobectomy. The surgical approach depended on surgeon choice and experience. All VATS cases were performed by one senior surgeon and open cases were operated on by other senior surgeons or trainees under supervision. Before surgery every patient underwent standard blood tests, chest computed tomography (CT) scan, bronchoscopy, abdominal ultrasound, pulmonary function tests and recently positron emission tomography-computed tomography (PET-CT) scan. Mediastinal lymph node enlargement above $15 \mathrm{~mm}$ on CT scan or positive lymph nodes in PET-CT were verified by mediastinoscopy. Patients with preoperatively confirmed positive N2 lymph nodes or after induction chemotherapy were excluded from this study. Perioperative outcomes including operating time, blood loss during surgery, postoperative complication rate, length of hospital stay, and duration of chest tube drainage were obtained through chart review. One hundred and eight patients underwent VATS lobectomy (VATS group) and 104 patients were operated on by antero-lateral muscle sparing thoracotomy, which is a standard open approach in our institution (thoracotomy group). The two groups of patients did not differ in terms of age, sex, tumor size, preoperative lung function tests and comorbidity rate. Patients' characteristics and perioperative findings are shown in Table I.

\section{Surgery and anesthesia}

Standard anesthesia technique including general anesthesia, single lung ventilation and thoracic epidural analgesia were used in all cases. Patients were placed in a lateral decubitus position with flexion of the operating table at the level of the tip of the scapula.

Our VATS technique consisted of three incisions: two ports and a 5-6 cm long utility incision without any kind of rib spreading. The whole of the procedure was controlled on the monitor via the thoracoscope. Two ports were placed in the seventh and eighth intercostal space in the anterior and posterior axillary line and were used for the thoracoscope and endoscopic instruments. The utility incision was performed in the midaxillary line in the fourth or fifth intercostal space and was used for endostaplers, endoscopic and standard instruments insertion and specimen withdrawal. Individual hilar dissection and ligation with endoscopic stapling devices followed by systematic mediastinal lymph node dissection were performed in every patient. All anatomical structures such as vessels, bronchus and lung parenchyma were closed and divided by endostaplers. In some cases semilock clips were used to close the smaller branches of the pulmonary artery in order to decrease the number of vascular reloads used. The sequence of dissection was different depending on the type of lobectomy and anatomical circumstances. Two chest tubes were inserted through the lower port incisions at the end of the procedure and were removed when 
Table I. Patients' characteristics

\begin{tabular}{|c|c|c|c|}
\hline Variable & VATS (108 patients) & Thoracotomy (104 patients) & Value of $p$ \\
\hline Age, mean \pm SD & $61.9 \pm 8.7$ & $61.6 \pm 7.9$ & 0.78 \\
\hline \multicolumn{4}{|l|}{ Gender, $n(\%)$ : } \\
\hline Female & $36(33)$ & $39(38)$ & 0.53 \\
\hline Male & $72(67)$ & $65(63)$ & \\
\hline Comorbidity rate: & $70(65)$ & $67(64)$ & 0.95 \\
\hline COPD, $n(\%)$ & $32(30)$ & $21(20)$ & 0.11 \\
\hline Diabetes, $n(\%)$ & $10(9)$ & $11(11)$ & 0.75 \\
\hline $\mathrm{CAD}, n(\%)$ & $17(16)$ & $18(17)$ & 0.76 \\
\hline Hypertension, $n$ (\%) & $32(30)$ & $37(36)$ & 0.35 \\
\hline \multicolumn{4}{|l|}{ Lung function tests: } \\
\hline Preop FEV ${ }_{1}[\%]$, mean \pm SD & $88 \pm 21$ & $86 \pm 21$ & 0.73 \\
\hline Preop VC [\%], mean \pm SD & $97 \pm 18$ & $92 \pm 19$ & 0.11 \\
\hline Tumor diameter, mean $[\mathrm{cm}]$ & $3.1 \pm 0.96$ & $3.3 \pm 1.2$ & 0.33 \\
\hline
\end{tabular}

$C A D$ - coronary artery disease, COPD - chronic obstructive pulmonary disease, FEV1 - forced expiratory volume in $1 \mathrm{~s}, \mathrm{VC}$ - vital capacity

there was no air-leak and fluid drainage was less than $150 \mathrm{cc}$ per $24 \mathrm{~h}$.

The open cases were operated on through anterolateral thoracotomy, sparing the latissimus dorsi muscle with standard rib spreading. Contrary to the thoracoscopic technique during the open procedure all the vessels were individually dissected and ligated by nonsoluble ligatures. No vascular endostaplers were used. Open linear staplers were used only to divide the lung parenchyma in the fissures. The bronchial stumps were closed by staplers or hand suture depending on the anatomic considerations and surgeon choice. Systemic mediastinal lymphadenectomy always followed lung resection. The procedure for chest tubes was the same.

The postoperative admission to the intensive care unit (ICU) depended on the patient's status during and after surgery and estimated risk of potential complications related to the patient's comorbidities. The patients were discharged home after chest tube removal with correct $X$-ray, without wound complications and when they could control the pain well and could cope independently in the home environment. The same discharge criteria were applied for both groups of patients.

\section{Cost evaluation}

Cost data were obtained for each individual patient after VATS and open resection from the hospital cost center and included: all direct theater costs related to the procedure, daily hospital costs, intensive care costs, pharmacy and disposable costs with special consideration of stapling device costs. Cost of buying the video-thoracoscope set and non-disposable surgical instruments and the costs of preoperative staging procedures have not been included in this analysis. All the direct medical costs of the procedure were calculated in Polish currency (zloty $-\mathrm{zl}$ ) and converted into Euro assuming $€ 1=4.0 \mathrm{zl}$ VAT was included.

\section{Statistical analysis}

Numerical data were expressed as means and standard deviations. If data did not follow a normal distribution data were presented as medians and interquartile ranges. Continuous variables were compared using two-tailed Student's $t$-test for independent samples, when both data were normally distributed (tested with the Shapiro-Wilk test) and homogeneity of variances was fulfilled (tested with 
Levene's test). If homogeneity of variance was not maintained alternatively Welch's test was used. If data were not normally distributed two-tailed nonparametric, Mann-Whitney $U$ test for unrelated variables was used. Dichotomous variables were compared using $\chi^{2}$ test of independence. $\mathrm{RxC}$ analyses of the categorical data were performed using $\chi^{2}$ test of independence or Fisher-Freeman-Halton exact test. All results were considered significant at $p<0.05$. Statistical analysis was performed using Statistica 9.0 by StatSoft and StatXact by CytelStudio 8 .

\section{Results}

The population analyzed was composed of $212 \mathrm{pa}-$ tients including 75 women and 137 men (mean age $61.7 \pm 8.3$ years, range $41-82$ years). One hundred and eight patients underwent VATS lobectomy (VATS group) and 104 lobectomy through antero-lateral muscle sparing thoracotomy (thoracotomy group). The majority of patients had non-small cell lung cancer (NSCLC) ( $n=205,97 \%)$. Adenocarcinoma (49\%) and squamous cell carcinoma (41\%) were the most common histological types of tumor. Stage I and II of resected lung cancer were predominant in both groups of patients (86\%). Stage IIIA lung cancer was significantly more common in the thoracotomy group. All the histopathological details are listed according to the approach chosen to perform the resection in Table II.

The mean hospital stay after VATS lobectomy was significantly shorter than after open lobectomy and was $7 \pm 3.4$ days vs. $10 \pm 6.5$ days $(p<0.0012$ ). The complication rate and ICU admission rate after surgery were almost twice as high after thoracotomy than after the thoracoscopic approach and were $46 \%$ vs. $23 \%(p<0.0006)$ and $42 \%$ vs. $22 \%(p<0.0027)$ respectively.

The mean duration of the surgery, median ICU stay and prolonged air leak rate after surgery did not differ between the VATS group and thoracotomy group. The median intraoperative blood loss in case of VATS resection was $50 \pm 75 \mathrm{ml}$ (range 25-600 ml) while the blood loss during conventional lobectomy was median $250 \pm 108 \mathrm{ml}$ (range 100-600 ml). This difference was also statistically significant

Table II. Histopathological details

\begin{tabular}{|c|c|c|c|}
\hline Variable & VATS & Thoracotomy & Value of $p$ \\
\hline \multicolumn{4}{|l|}{ Diagnosis, $n(\%)$ : } \\
\hline NSCLC & $104(96)$ & $101(97)$ & \\
\hline Benign & $4(4 \%)$ & $3(3 \%)$ & \\
\hline \multicolumn{4}{|c|}{ NSCLC histology, $n$ (\%): } \\
\hline Adenocarcinoma & $51(49)$ & $42(42)$ & 0.29 \\
\hline SCC & $43(41)$ & $48(47)$ & 0.37 \\
\hline Carcinoid & $3(3)$ & $5(5)$ & 0.44 \\
\hline Other & $7(7)$ & $6(6)$ & 0.82 \\
\hline NSCLC stage, $n(\%)$ : & & & 0.0796 \\
\hline Stage IA & $40(38)$ & $28(28)$ & \\
\hline Stage IB & $38(37)$ & $28(28)$ & \\
\hline Stage IIA & $14(13)$ & $23(23)$ & \\
\hline Stage IIB & $3(3)$ & $4(4)$ & \\
\hline Stage IIIA & $7(7)$ & $16(16)$ & $0.0433^{*}$ \\
\hline Stage IV & $2(2)$ & $2(2)$ & \\
\hline
\end{tabular}

NSCLC - non-small cell lung cancer, SCC - squamous cell carcinoma, *significantly different 
Table III. Clinical data

\begin{tabular}{|lccc|}
\hline Variable & VATS & Thoracotomy & Value of $p$ \\
\hline Hospital stay, mean \pm SD [days] & $7 \pm 3.4$ & $10 \pm 5.5$ & 0.0012 \\
\hline Complication rate, $n(\%)$ & $25(23)$ & $47(46)$ & 0.0006 \\
\hline ICU admission rate, $n(\%)$ & $24(22)$ & $44(42)$ & 0.0027 \\
\hline ICU stay, mean \pm SD, range [days] & $4 \pm 2.86,1-16$ & $4 \pm 2.16,1-13$ & $0.14^{*}$ \\
\hline Drainage time, median, range [days] & $3,2-14$ & $4,3-24$ & 0.0043 \\
\hline Prolonged air leak (over 5 days), $n(\%)$ & $15(16)$ & $22(21)$ & $0.22^{*}$ \\
\hline Time of surgery, mean \pm SD, range [min] & $128 \pm 35,55-240$ & $133 \pm 37,65-240$ & $0.25^{*}$ \\
\hline Intraoperative blood loss, median, range [ml] & $50(75), 25-600$ & $250(108), 100-600$ & 0.0001 \\
\hline
\end{tabular}

${ }^{*} N S$

Table IV. Surgical procedures and stapler consumption

\begin{tabular}{|lcccc|}
\hline Type of surgery & VATS $(n)$ & Thoracotomy $(n)$ & $\begin{array}{c}\text { VATS reload number, } \\
\text { mean } \pm \text { SD }\end{array}$ & $\begin{array}{c}\text { Thoracotomy reload number, } \\
\text { mean } \pm \text { SD }\end{array}$ \\
\hline RUL & 41 & 37 & $7.5 \pm 3.0$ & $3.1 \pm 1.4$ \\
\hline LUL & 30 & 28 & $6.1 \pm 1.9$ & $2.4 \pm 1.3$ \\
\hline RLL & 21 & 18 & $6.8 \pm 1.9$ & $2.6 \pm 1.1$ \\
\hline LLL & 11 & 18 & $5.2 \pm 2.5$ & $2.3 \pm 1.8$ \\
\hline LM & 5 & 3 & $4.0 \pm 1.5$ & $2.0 \pm 1.0$ \\
\hline
\end{tabular}

LLL - left lower lobectomy, LM - middle lobectomy, LUL - left upper lobectomy, RLL - right lower lobectomy, RUL - right upper lobectomy

$(p<0.0001)$ (Table III). Most patients underwent upper lobectomy in both the VATS and thoracotomy group (66\% and $72 \%)$. The details of performed surgical procedures are shown in Table IV.

Cost analysis showed considerably higher theater costs for the VATS group compared to the thoracotomy group, median $€ 1395$, range from $€ 632$ to $€ 3388$ vs. median $€ 479$, range from $€ 127$ to $€ 1374$. This difference was caused mainly by endostapler costs used during the thoracoscopic approach. The stapler costs in the VATS group were over five times higher than in the thoracotomy group and the median was $€ 1069$ vs. $€ 161$ respectively. Significantly higher hospital costs and ICU costs of patients after open lobectomy did not compensate high theater costs of VATS lobectomy. Finally, total costs of VATS lobectomy in our group of patients and Polish economic conditions were significantly higher than after thoracotomy and the median was $€ 2445$ compared to $€ 2047$. All the financial details are shown in Tables $\mathrm{V}$ and $\mathrm{VI}$.

\section{Disscusion}

It has been shown by many published reports that the VATS approach has some advantages over conventional rib-spreading thoracotomy in treatment of patients with early stage NSCLC and other thoracic pathologies [10]. They are as follows: less postoperative pain, shorter hospitalization, fewer postoperative complications, better tolerance of adjuvant chemotherapy with comparable long-term survival rate [11]. Our results confirmed the advantages of VATS lobectomy over an open procedure in terms of shorter hospital stay, lower postoperative complication rate, smaller intraoperative blood loss and lower ICU admission rate. The ICU admission rate was twice as high after thoracotomy and reached $42 \%$ compared to VATS $22 \%$. It has to be stated that such a high general ICU admission rate not only reflects the number of severe postoperative complications but was the result of insufficient intermediate care at our department. 
Table V. Cost analysis

\begin{tabular}{|lccc|}
\hline Variable & VATS $(n=108)$ & Thoracotomy $(n=104)$ & Value of $p$ \\
\hline Theater costs, median (IQR), range $[€]$ & $1395(662), 632-3388$ & $479(250), 127-1374$ & 0.0001 \\
\hline Stapler costs, median (IQR), range $[€]$ & $1069(648), 307-3202$ & $161(145)$, range 0-1129 & 0.0001 \\
\hline Hospital stay costs, median (IQR), range [€] & $700(200), 400-2500$ & $1000(750), 600-5400$ & 0.0001 \\
\hline ICU costs, median (IQR), range [€] & $930(284), 0-5374$ & $1000(483), 0-3033$ & 0.0352 \\
\hline Total costs, median (IQR), range [€] & $2445(953), 800-9144$ & $2047(1213), 746-7962$ & 0.0046 \\
\hline
\end{tabular}

Table VI. Type of staplers and costs

\begin{tabular}{|c|c|}
\hline Staplers & Cost $[€]$ \\
\hline \multicolumn{2}{|l|}{ VATS } \\
\hline Endostapler Endo GIA Uni ${ }^{1}$ & 275 \\
\hline Endo GIA Uni Rot cartridge 45 mm 1 & 160 \\
\hline DUET TRS cartridge $60 \mathrm{~mm}^{1}$ & 266 \\
\hline Endostapler Multifire Endo GIA 30 mm² & 160 \\
\hline SCB 45 cartridge 2 & 85 \\
\hline \multicolumn{2}{|l|}{ Thoracotomy } \\
\hline Linear stapler 60 mm³ & 93 \\
\hline Linear stapler cartridge $60 \mathrm{~mm}^{3}$ & 68 \\
\hline Linear stapler GIA $(60 \mathrm{~mm}, 80 \mathrm{~mm})^{3}$ & 125 \\
\hline Linear stapler GIA cartridge LCR 80G 3 & 55 \\
\hline Linear stapler GIA cartridge LCR 60G 3 & 35 \\
\hline $\begin{array}{l}\text { Linear stapler cartridge } 30 \mathrm{~mm} \\
\text { (for non-disposable stapler) }\end{array}$ & 49 \\
\hline
\end{tabular}

${ }^{1}$ Covidien, ${ }^{2}$ Ethicon, Johnson\&Johnson, ${ }^{3}$ Beryl Med Ltd.

Despite confirmed advantages, VATS lobectomy still remains a relatively infrequently performed procedure [12]. There are not so many centers where significant groups of patients with resectable lung cancer are operated on by this minimally invasive approach. According to the Society of Thoracic Surgeons database from 1999 to 2006 only 20\% of all lobectomies for lung cancer were performed thoracoscopically in the United States [13]. Rocco et al. published in 2008 a very interesting report based on an internet survey among mostly the European Society of Thoracic Surgeons (ESTS) members regarding the use of VATS technique in their clinical practice [14]. The results showed that VATS lobectomy was performed by $49 \%$ of responders but as many as $75 \%$ of them declared the rate of minimally invasive procedures compared to open below $5 \%$. Only $15 \%$ of responders declared that in over $30 \%$ of all patients with early stage lung cancer VATS was used.

In Poland, the first thoracoscopic lobectomy was performed at the Department of Thoracic Surgery of Poznan University of Medical Sciences in 1999, but until 2006 less than 3\% of patients treated in our center were operated on using this technique. During the next 3 years the rate of VATS lobectomies increased up to 17\% in 2009 and 25\% in 2010 and is still increasing. Until 2009 our department was the only one where the VATS lobectomy was routinely performed in Poland [15]. The total number of resections due to lung cancer in Poland in 2009 reached over 3000 cases and only 3\% were performed by VATS.

Many factors may influence this situation. The most important and frequently quoted are: new surgical technique, demanding training, steep learning curve and logistic and economic issues. Factors such as new surgical technique, difficulties in optimal training and steep learning curve are common for everyone who starts a VATS lobectomy program [16, 17]. On the other hand, the economic and logistic issues related to the procedure vary depending on countries with different national income. The differences in local health care systems and the reimbursement systems of the medical procedures also have a strong impact on the results. There are only a few reports in the literature analyzing the economic costs of VATS lobectomy compared to open technique [7-9]. All of them come from high income countries and reflect the economic reality in the USA, UK or Japan. Most of these studies showed significantly higher direct theater costs of VATS lobectomy compared to the open procedure and the endostapler costs have been the biggest part of them. This is related to the surgical technique which consists of individual hilar dissection 
and ligation with endoscopic stapling devices. The same technique was used in our institution.

Casali and Walker reported twice as high theater costs of VATS lobectomy compared to thoracotomy. These higher costs were also related to endostaplers used during resection [8].

In contrast to these results Burfeind Jr and D'Amico showed that in their economic reality costs for the hospitalization, including theater costs, were less for the VATS lobectomy than the open procedure. The results could be explained by the fact that the authors used the same endostaplers during VATS and thoracotomy [7]. Furthermore, Park and Flores showed than even robotic VATS lobectomy with the da Vinci system can be less expensive than the open approach in American reality [18].

Our cost analysis clearly showed considerably higher theater costs of VATS lobectomy compared to open thoracotomy (€1395 vs. €479). All theater costs were almost three times higher in the VATS group and stapler costs were over six times higher ( $€ 1069$ vs. €161) compared to thoracotomy. This enormous disproportion resulted from completely different surgical technique used during thoracotomy when all vessels are ligated by nonabsorbable sutures and no vascular endostaplers are used. The linear staplers were used only to close the bronchus and to divide the fissure. The costs and the number of open staplers used during thoracotomy were significantly lower than costs of endostaplers used during minimally invasive resection.

Apart from higher costs of the procedure all authors underline important clinical advantages of the minimally invasive approach such as fewer postoperative complications, faster recovery, lower ICU admission rate, shorter HDU stay and finally shorter postoperative hospital stay. These benefits of the VATS approach could decrease general costs of treatment and compensate higher costs of the surgery in some circumstances. Final costs of VATS lobectomy would be equal to or even less than after thoracotomy.

In the paper from Edinburgh by Casali and Walker it was clearly shown that twice as high theater costs of VATS lobectomy were compensated by higher HDU and ward stay costs after thoracotomy. The total costs of treatment were higher after thoracotomy than after the thoracoscopic approach. Burfeind Jr reported similar economic benefits of VATS lobectomy related to shorter hospital stay.
Nakajima et al. also reported lower total costs of VATS lobectomy compared to thoracotomy in Japan [19]. However, it should be noted that the hospital stay was very long in their group of patients and the mean was 17.3 days after thoracoscopy and 23.8 days after thoracotomy, which is unusual in Europe.

In Polish financial reality very high theater costs of VATS lobectomy were not compensated by significantly lower costs of shorter postoperative hospital stay, lower ICU admission rate, and faster recovery. At the time of the study the cost of 1-day hospital stay in Poland was very low compared to the data published by other authors and the amount is about $€ 100$ per day. It results in significantly higher total costs of VATS lobectomy compared to thoracotomy in Polish economic circumstances. On the other hand, a reimbursement from the NHF is paid for the procedure and the amount is the same for open and VATS lobectomy.

The shorter hospital stay after VATS lobectomy potentially allows an increase of the number of procedures for the same number of beds in the ward. It can give additional profits but only in the situation when there are no limits of procedures in hospital. In Poland it is not possible because every hospital has a contract with the National Health Fund (NHF) for the number of procedures which are reimbursed.

It has to be stated that our study has some limitations. First, it is not a prospective randomized but a retrospective study. Nevertheless, it is very difficult to randomize patients when the surgeon is convinced about the clinical benefits of the VATS approach over thoracotomy so having two groups of surgeons was the only way to obtain two groups. The patients in the VATS and thoracotomy group did not differ in terms of age, sex, lung cancer stage and comorbidities. Costs of medical staff and economic benefits related to faster recovery after surgery also were not calculated - first because the medical staff salaries had no relation to the type of the surgery; and second, the economic benefits related to faster recovery and faster return to work were very difficult to calculate.

\section{Conclusions}

The results confirmed many clinical benefits for patients after VATS lobectomy compared to thoracotomy. The cost analysis however showed significant- 
ly higher total costs of VATS lobectomy due to essentially higher theater costs in Poland and significant lower costs of hospitalization. One has to be very cautious in drawing any general conclusion because the economic results depend very much on the local fund system of medical procedures and the results can completely differ. We are convinced that the presented results can have an important value in the general debate about the costs of VATS lobectomy.

We assume that Polish financial conditions do not substantially differ from the economic conditions in other middle-income countries and that the financial aspect of the procedure can limit widespread introduction of VATS lobectomy in clinical practice in these countries. Nowadays, when we have to face the worldwide financial crisis, this economic aspect of VATS lobectomy can be additionally very important worldwide.

\section{References}

1. Jemal A, Siegel R, Ward E, et al. Cancer statistics, 2008. CA Cancer J Clin 2008; 58: 71-96.

2. Ettinger DS, Akerley W, Bepler G, et al. National Comprehensive Cancer Network (NCCN). Non-small cell lung cancer clinical practice guidelines in oncology. I Natl Compr Cancer Network 2008; 6: 228-69.

3. Onaitis MW, Petersen PR, Balderson SS, et al. Thoracoscopic lobectomy is a safe and versatile procedure: experience with 500 consecutive patients. Ann Surg 2006; 244: 420-5.

4. McKenna RJ, Houck W, Fuller CB. Video-assisted thoracic surgery lobectomy: experience with 1100 cases. Ann Thorac Surg 2006; 81: 421-6.

5. Swanson SJ, Herndon JE, D'Amico TA, et al. Video-assisted thoracic surgery (VATS) lobectomy-Report of CALGB 39802: a prospective, multi-institutional feasibility study. I Clin Oncol 2007; 25: 4993-7.

6. Craig SR, Leaver HA, Yap PL, et al. Acute phase responses following minimal access and conventional thoracic surgery. Eur J Cardiothorac Surg 2001; 20: 455-63.

7. Burfeind WR, Jaik NP, Villamizar N, et al. A cost-minimisation analysis of lobectomy: thoracoscopic versus posterolateral thoracotomy. Eur J Cardiothorac Surg 2010; 37: 827-32.

8. Casali G, Walker WS. Video-assisted thoracic surgery lobectomy: can we afford it? Eur J Cardiothorac Surg 2009; 35: 423-8.

9. Walker WS, Casali G. The VATS lobectomy: analysis of costs and alterations in the traditional surgical working pattern in the modern surgical unit. Thorac Surg Clin 2008; 18: 281-7.

10. Neoral C, Aujeský R, Škarda J, et al. Thoracoscopic treatment of benign esophageal tumors. Videosurgery Miniinv 2012; 7: 294-8.

11. Petersen RP, Pham D, Burfeind WR, et al. Thoracoscopic lobectomy facilitates the delivery of chemotherapy after resection for lung cancer. Ann Thorac Surg 2007; 83: 1245-9.

12. Duda M, Gryga A, Czudek S, Skalický P. Twenty years of minimally invasive surgery in the Czech Republic. Videosurgery Miniinv 2011; 6: $42-7$.
13. Boffa DJ, Allen MS, Grab JD, et al. Data from The Society of Thoracic Surgeons General Thoracic Surgery database: the surgical management of primary lung tumors. J Thorac Cardiovasc Surg 2008; 135: 247-54

14. Rocco G, Internullo E, Cassivi SD, et al. The variability of practice in minimally invasive thoracic surgery for pulmonary resections. Thorac Surg Clin 2008; 18: 235-47.

15. Piwkowski C, Gabryel P, Kasprzyk M, Dyszkiewicz W. Videoassisted thoracic surgery pneumonectomy: the first case report in Poland. Videosurgery Miniinv 2012; 7: 197-201.

16. McKenna RJ. Complications and learning curves for video-assisted thoracic surgery lobectomy. Thorac Surg Clin 2008; 18: 275-80.

17. Petersen RH, Hansen HJ. Learning thoracoscopic lobectomy. Eur J Cardiothorac Surg 2010; 37: 516-20.

18. Park BJ, Flores RM. Cost comparison of robotic, video assisted thoracic surgery and thoracotomy approaches to pulmonary lobectomy. Thorac Surg Clin 2008; 18: 297-300.

19. Nakajima J, Takamoto S, Kohno T, Ohtsuka T. Costs of videothoracoscopic surgery versus open resection for patients with lung carcinoma. Cancer 2000; 89: 2497-501.

Received: 18.02.2013, accepted: 28.04.2013. 